\title{
Pulsed supersonic jet epitaxy: A nonthermal approach to silicon growth
}

\author{
Rajeev Malik and Erdogan Gularia) \\ Department of Chemical Engineering and Center for Display Technology and Manufacturing, \\ University of Michigan, Ann Arbor, Michigan 48109
}

(Received 11 January 1996; accepted for publication 23 March 1996)

\begin{abstract}
In this letter, we demonstrate a unique approach for low temperature epitaxial growth of single crystal silicon films on $\mathrm{Si}(100)$. Pulsed supersonic jet epitaxy (PSJE), employs high kinetic energy jets of a disilane-hydrogen mixture incident on the surface leading to layer by layer growth. Precise control of film thickness and significantly higher sticking coefficients are demonstrated. Growth rate dependence of pulse frequency and its implications are discussed. We have been able to reproducibly deposit good quality single crystalline films at temperatures as low as $400{ }^{\circ} \mathrm{C}$ with this technique, without the use of any external activation. (c) 1996 American Institute of Physics.
\end{abstract} [S0003-6951(96)03022-7]

Low temperature processing is of primary importance with the ever decreasing size of integrated circuits and devices in today's semiconductor industry. Thermally activated processes like dopant diffusion need to be strictly controlled to obtain abrupt interfaces in multilayer structures. Flat panel displays employ glass substrates which undergo softening above about $550{ }^{\circ} \mathrm{C}$. As a result, thin film transistors (TFTs) have to be fabricated with amorphous silicon which suffers from relatively poor device performance. This has necessitated reducing the thermal budget for growth and looking for alternatives. Conventional growth processes employing hydridic precursors, however, are not effective at low temperatures due to surface hydrogen desorption limitations. The primary focus in this work is to utilize the high translational energy associated with a supersonic jet rather than substrate thermal energy to overcome activation barriers.

Supersonic molecular beams have conventionally been employed along with spectroscopic detection techniques to elucidate the gas-surface dynamics of various systems. ${ }^{1,2}$ As opposed to an effusive beam, which has a broad MaxwellBoltzmann energy distribution and low kinetic energy, supersonic beams possess a very narrow velocity distribution and hence produce a fairly monoenergetic flux of molecules. Also, source energies over a wide range of $\mathrm{eV}$ can be obtained by employing a mixture of the precursor gas and a light carrier gas (seeding). ${ }^{3}$ The versatility of supersonic beams has been demonstrated in digital etching, ${ }^{4}$ growth, ${ }^{5-10}$ and been proposed as potentially important in atomic layer epitaxy. ${ }^{11}$

Silicon growth from hydride precursors has been extensively reported in literature. Kinetics studies indicate ${ }^{12}$ that growth proceeds via a precursor mediated mechanism, i.e., the incident molecule is first physisorbed ("trapped") and only a fraction of these undergo chemisorption. Disilane $\left(\mathrm{Si}_{2} \mathrm{H}_{6}\right)$, which is the preferred precursor has an inherent disadvantage due to its hydridic nature. The film formation process is strongly dependent on the concentration of non-Hdepleted sites on the surface which in turn is temperature driven. ${ }^{13}$ Hence, the motivation here is to overcome the activation barrier for the H-removal process but still maintain

a)Electronic mail: gulari@engin.umich.edu the substrate temperature within limits. In this letter, we report silicon epitaxy at low temperatures using supersonic jets of disilane. We have not only obtained good quality single crystal silicon at $400{ }^{\circ} \mathrm{C}$ with comparable growth rates but also been able to demonstrate precise growth control.

The experiments were conducted in a RIBER gas source MBE 32 system modified to incorporate a pulsed supersonic valve (General Valve Series 9, $0.7 \mathrm{~mm}$ nozzle) with an electronic controller. ${ }^{14}$ The pulse width (open time) was varied from 10 to $100 \mathrm{~ms}$. The pulse frequency was varied from 0.08 to $0.33 \mathrm{~Hz}$ (pulse operation gives an additional control parameter to regulate the amount of material delivered to the sample and also helps to ensure a certain vacuum in the growth chamber due to the high flux generated with this technique). A $2 \%$ disilane in hydrogen mixture (Voltaix, Inc.) was used as a source gas from a reservoir maintained at a pressure of $1.1 \mathrm{~atm}$. The resultant beam energy (with a room temperature nozzle) is equivalent to about $1.2 \mathrm{eV}$ based on calculations and data reported in literature. ${ }^{15,16}$ The base pressure in the MBE chamber before growth was in the $10^{-9}$ Torr range; the peak beam pressure during growth was about $10^{-4}$ Torr. The $p$-type $\mathrm{Si}$ (100) wafers (10-20 $\Omega \mathrm{cm})$ were degreased followed by a $30 \mathrm{~s} \mathrm{HF}(10 \%)$ etch and a $\mathrm{N}_{2}$ dry before being loaded. Prior to growth, the sample was heated to about $800{ }^{\circ} \mathrm{C}$ to desorb the native oxide. A buffer silicon epitaxial layer was grown by conventional GSMBE to bury any existing impurities and ensure a smooth starting surface as observed by reflection high energy electron diffraction (RHEED). A monolayer of germanium was deposited on all samples to act as a marker layer to distinguish the film from the substrate. Cross-sectional transmission electron microscopy (XTEM) and glancing angle Rutherford backscattering spectrometry (GA-RBS) were used to determine film thicknesses and crystallinity. Growth studies were performed at temperatures between 400 and $500{ }^{\circ} \mathrm{C}$.

Pulse width, pulse frequency, and substrate temperature were some of the parameters used to characterize this system. The error bars reported in all figures arise from uncertainty in GA-RBS thickness measurements and comparison with XTEM data from some of the films. Also, all growth rates (GR) have been computed on a "per pulse"' basis due to the noncontinuous nature of the experiment. Figure 1 


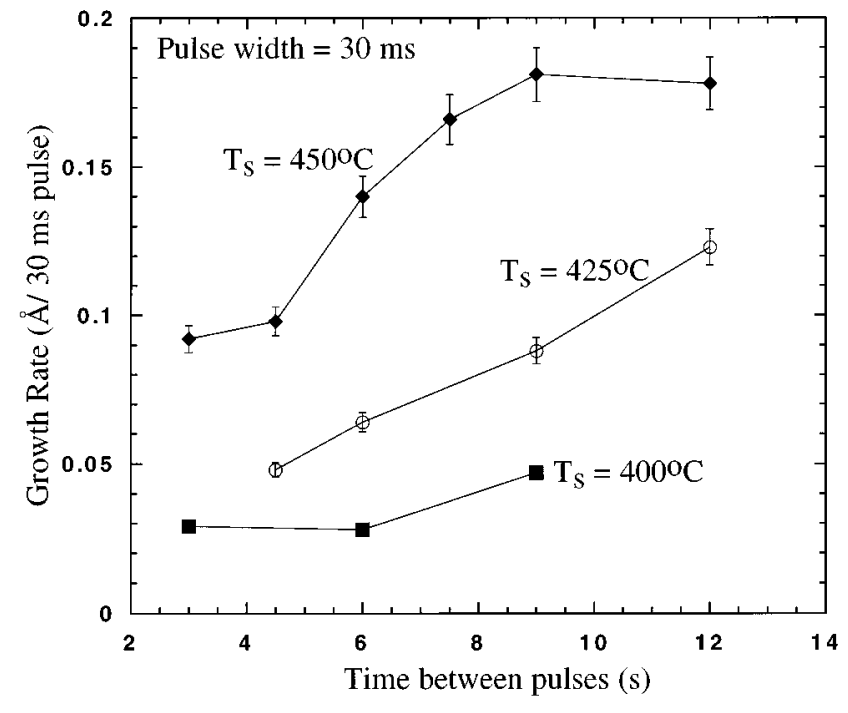

FIG. 1. Silicon growth rate in $\AA$ /pulse for a $30 \mathrm{~ms}$ pulse, as a function of the time allowed between successive pulses (s). The lines connecting the points are only meant to guide the eye.

shows how the GR varies as a function of the time allowed between successive pulses. The latter turns out to be a crucial parameter controlling the amount of material incorporated per pulse. This "off time" is essentially the time allowed for the surface to regenerate sites which are hydrogen terminated immediately following chemisorption of the precursor. The hydrogen desorption process hence controls the growth in this temperature regime. Its time-temperature dependent nature is evident from the fact that at $T=450{ }^{\circ} \mathrm{C}$, increasing the time for desorption beyond $9 \mathrm{~s}$ does not produce any increase in the growth rate implying that a "quasiequilibrium" has been reached. We can also see that at higher temperatures, this "saturation" point would move to the left in the plot eventually allowing continuous operation to be the most efficient. Figure 2 shows an Arrhenius plot for pulsed growth of silicon in the $400-500{ }^{\circ} \mathrm{C}$ range for the two extreme cases

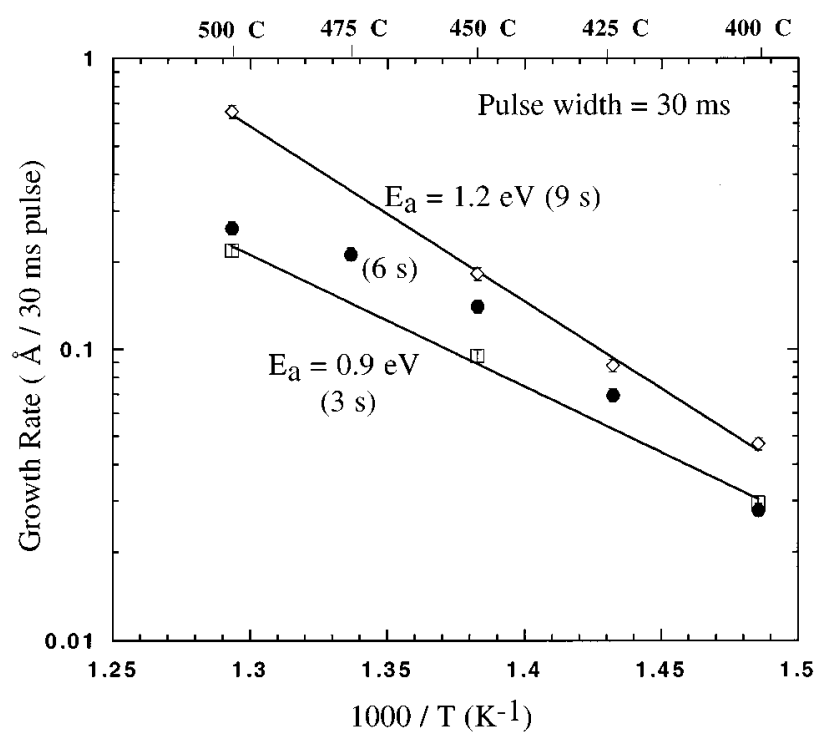

FIG. 2. Arrhenius plot of the film growth rate (in $\AA /$ pulse) with a $30 \mathrm{~ms}$ pulse for 3, 6, and $9 \mathrm{~s}$ between pulses. The fit for the $6 \mathrm{~s}$ case (filled circles) is not shown for clarity.

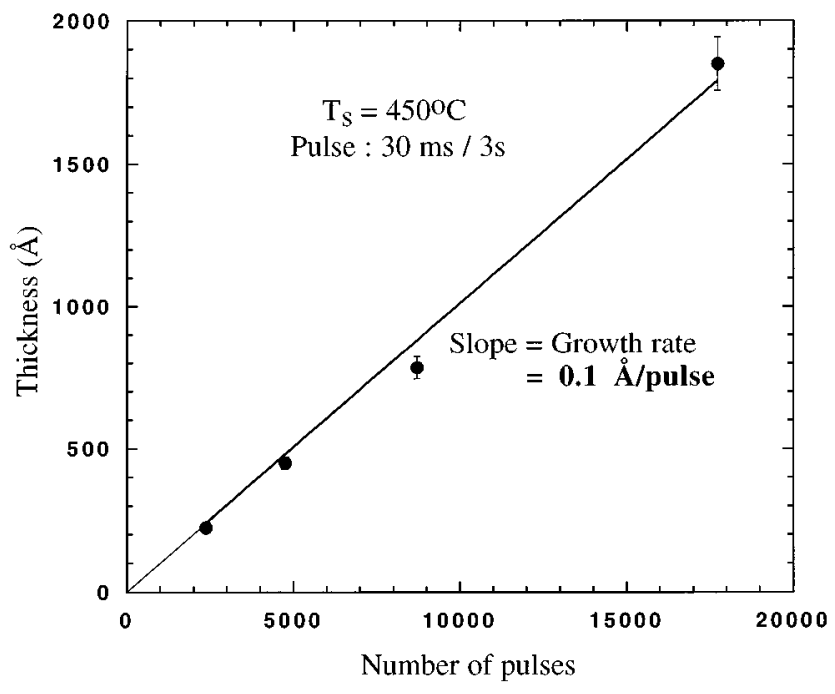

FIG. 3. Silicon film thickness in $\AA$ as a function of the total number of pulses delivered.

of 3 and $9 \mathrm{~s}$ between pulses. In this temperature range (see Fig. 1), we believe that $3 \mathrm{~s}$ is short enough that thermal desorption processes are not dominant and the low activation energy can be attributed to collision induced desorption (promoting surface recombination of $\mathrm{H}$ atoms purely due to impact of incident molecule). On the other hand, $9 \mathrm{~s}$ is a relatively large time interval and this case represents an $\mathrm{E}_{\mathrm{a}}$ dominated by thermal hydrogen desorption. The $6 \mathrm{~s}$ case is in an intermediate regime. The activation energies can hence be interpreted as "lumped parameters" which include effects of the two kinds of desorption processes with varied substrate temperature dependencies (the chemisorption process itself is known to be unactivated).

An important result to emerge from this study is shown in Fig. 3. Films grown under the same conditions but for different total times (i.e., total number of gas pulses) not only show striking reproducibility but also exhibit a linear variation of the growth rate. The implication of this finding is that precise thickness control at the submonolayer level is achieved by simply regulating the total number of pulses delivered to the substrate. This has potential applications in atomic layer epitaxy (ALE), a process suitable for growth of very thin films in a layer by layer mode. Our process can more aptly be termed as digital epitaxy ${ }^{17}$ due to the fact that less than $1 \mathrm{ML}$ is formed per cycle. Similar behavior is seen for other conditions of pulse widths and temperatures.

We have also conducted a comparison study of this technique with conventional gas source MBE. Sticking probabilities (S), defined as the fraction of incident molecules incorporated in the film, for the two methods were calculated (accounting for growth in the "off time" of the pulse due to the background). It was found that PSJE had more than an order of magnitude higher $\mathrm{S}$ values than GSMBE. $\mathrm{S}$ was about 0.065 at $400{ }^{\circ} \mathrm{C}$ and 0.15 at $450{ }^{\circ} \mathrm{C}$ for our technique. The latter value is in good agreement with data reported at high temperatures using rigorous surface science studies. ${ }^{15} \mathrm{~A}$ practical implication of higher sticking probabilities is in the efficient utilization of precursors resulting in better economic performance. 


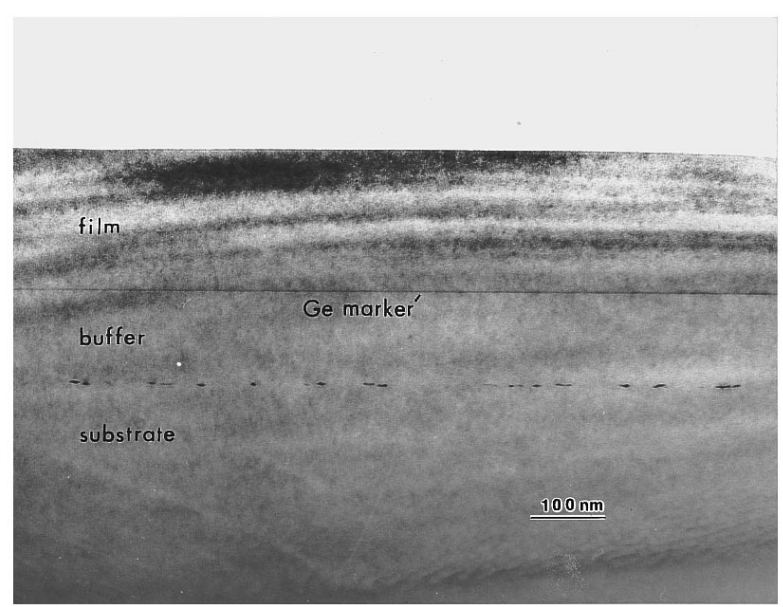

FIG. 4. Cross section TEM of a film grown at $450{ }^{\circ} \mathrm{C}$ with $30 \mathrm{~ms} / 6 \mathrm{~s}$ pulse for a total of $900 \mathrm{~min}$. The film (between the interface and the Ge marker) is about $1850 \AA$ thick.

Low temperature growth studies of silicon homoepitaxy using gas sources in literature have resorted to external activation by either synchrotron radiation ${ }^{18}$ or lasers. ${ }^{19,20}$ The mechanism behind these processes, however, involves complexities owing to gas phase as well as surface interactions. PSJE, being a surface acting method simplifies the growth process by its fundamental approach. Figure 4 shows a typical XTEM micrograph of an epitaxial silicon film grown at $450{ }^{\circ} \mathrm{C}$. The thickness of the film obtained was $\sim 1850 \AA$ with 17700 pulses of duration $30 \mathrm{~ms}$ each, translating into a GR of about $0.1 \AA$ Apulse. Even if the total growth time was taken into consideration, a growth rate of $2 \AA / \mathrm{min}$ is obtained which is comparable to values in literature at these low temperatures. ${ }^{19}$ High resolution TEM of the films grown at $400{ }^{\circ} \mathrm{C}$ confirm crystallinity and show no significant defects in the silicon epilayer. ${ }^{21}$

In conclusion, low temperature epitaxial growth of silicon from gas sources has been successfully demonstrated. Supersonic jets are shown to be an effective nonthermal medium in providing precursor activation. We believe that they not only help in dissociating the disilane molecule but also aid in removal of the hydrogen by collision-induced desorp- tion, a phenomenon observed in other systems. ${ }^{1}$ By increasing the incident energy to about $2 \mathrm{eV}$ (the activation energy for hydrogen desorption), we expect to be able to further lower the minimum temperature for single crystal silicon growth. This possibility will be pursued by heating the pulsed nozzle. We have shown the ability of PSJE to conduct digital epitaxy which is a promising technique having applicability in thin film processes requiring accurate thickness control.

This work was supported by a grant from the Air Force Office of Scientific Research (AFOSR) and the Center for Display Technology and Manufacturing (DTM) at the University of Michigan.

${ }^{1}$ S. T. Ceyer, Annu. Rev. Phys. Chem. 39, 479 (1988).

${ }^{2}$ J. B. Anderson, in Molecular Beams and Low Density Gas Dynamics (Marcel Dekker, New York, 1974), p. 1.

${ }^{3}$ N. Abuaf, J. B. Anderson, R. P. Andres, J. B. Fenn, and D. G. H. Marsden, Science 155, 997 (1967).

${ }^{4}$ Y. Teraoka and I. Nishiyama, Appl. Phys. Lett. 63, 3355 (1993).

${ }^{5}$ S. S. Lee, D. W. Minsek, D. J. Vestyk, and P. Chen, Science 263, 1596 (1994).

${ }^{6}$ D. Wang, T.-P. Ma, J. W. Golz, B. L. Halpern, and J. J. Schmitt, IEEE Electron Device Lett. 13, 482 (1992).

${ }^{7}$ T. Ohmi, M. Morita, T. Kochi, M. Kosugi, H. Kumagai, and M. Itoh, Appl. Phys. Lett. 52, 1173 (1988).

${ }^{8}$ K.-J. Kim, M. Suemitsu, and N. Miyamoto, J. Vac. Sci. Technol. 12, 986 (1994).

${ }^{9}$ D. Eres, D. H. Lowndes, J. Z. Tischler, J. W. Sharp, T. E. Haynes, and M. F. Chisholm, J. Appl. Phys. 67, 1361 (1990).

${ }^{10}$ S. Zhang, J. Cui, A. Tanaka, and Y. Aoyagi, Appl. Phys. Lett. 64, 1105 (1994).

${ }^{11}$ M. Yoder, Thin Solid Films 225, 145 (1993).

${ }^{12}$ M. L. Yu and L. A. DeLouise, Surf. Sci. Rep. 19, 285 (1994).

${ }^{13}$ S. M. Gates and C. M. Chiang, Chem. Phys. Lett. 184, 448 (1991).

${ }^{14}$ R. Malik, E. Gulari, S. H. Li, P. K. Bhattacharya, and J. Singh, J. Cryst. Growth 150, 984 (1995).

${ }^{15}$ D. R. Miller in Atomic and Molecular Beam Methods, edited by G. Scoles (Oxford University Press, New York, 1988), p.14.

${ }^{16}$ J. R. Engstrom, D. A. Hansen, M. J. Furjanic, and L. Q. Xia, J. Chem. Phys. 99, 4051 (1993).

${ }^{17}$ G. Eres and J. W. Sharp, J. Appl. Phys. 74, 7241 (1993).

${ }^{18}$ Y. Nara, Y. Sugita, K. Horiuchi, and T. Ito, Appl. Phys. Lett. 61, 93 (1992).

${ }^{19}$ B. Fowler, S. Lian, S. Krishnan, L. Jung, C. Li, D. Samara, I. Manna, and S. Banerjee, J. Appl. Phys. 72, 1137 (1992).

${ }^{20}$ D. Lubben, R. Tsu, T. R. Bramblett, and J. E. Greene, J. Vac. Sci. Technol. 9, 3003 (1991).

${ }^{21}$ R. Malik and E. Gulari (unpublished). 